\title{
EL DESARROLLO DE PERÍFRASIS INCOATIVAS CUASI-SINÓNIMAS: ENTRE CONSTRUCCIONALIZACIÓN Y LEXICALIZACIÓN
}

\section{THE DEVELOPMENT OF NEARLY-SYNONYMOUS INCHOATIVE PERIPHRASES: BETWEEN CONSTRUCTIONALIZATION AND LEXICALIZATION}

\author{
RENATA ENGHELS \\ Ghent University \\ Renata.Enghels@UGent.be \\ SVEn VANHULle \\ Ghent University \\ Sven.Vanhulle@UGent.be
}

Enviado: $15 / 01 / 2018$

Aceptado: 07/09/2018

\begin{abstract}
Resumen
Este estudio se concentra en la evolución de las perífrasis incoativas en español. En estas construcciones, verbos de colocación (ponerse), de cambio (romper) o de movimiento (echarse) funcionan como auxiliar, combinándose con un infinitivo subordinado al que añade significado aspectual. El artículo pretende (i) analizar el origen y la evolución funcional y formal de una variante poco estudiada, romper $a+$ infinitivo, (ii) fijar con más precisión el tipo de cambio lingüístico que ilustra, lexicalización o construccionalización, y (iii) contestar a la pregunta de hasta qué punto perífrasis cuasi-sinónimas se han desarrollado en paralelo. Se exponen los resultados de un análisis comparativo diacrónico de tres perífrasis incoativas entre los siglos XIII y XXI. Está demostrado que el esquema incoativo se ha desarrollado a partir de verbos de co-
\end{abstract}

\begin{abstract}
This study deals with the evolution of inchoative periphrases in Spanish. In these constructions, verbs of placement (ponerse), change (romper) or movement (echarse) function as auxiliary verbs, combining with a subordinate infinitive to which they add aspectual meaning. The paper aims (i) to analyze the origins, as well as the functional and formal evolution of an understudied variant, romper $a+$ infinitive, (ii) to determine the type of process of linguistic change it illustrates, lexicalization or constructionalization, and (iii) to answer the question to what extent near-synonymous periphrases develop in parallel ways. It exposes the results of a comparative diachronic study of three periphrases between the $13^{\text {th }}$ and $21^{\text {st }}$ centuries. It is shown that the inchoative schema has developed from placement verbs and, only in a later phase, included other
\end{abstract}

Para citar este artículo / To cite this article: Enghels, Renata y Sven Vanhulle (2018). El desarrollo de perífrasis incoativas cuasi-sinónimas: entre construccionalización y lexicalización. ELUA, 32: 91110. doi: 10.14198/ELUA2018.32.4

Enlace / Link: http://dx.doi.org/10.14198/ELUA2018.32.4 
locación y que posteriormente se abre a incluir otros tipos léxicos. Para explicar el proceso de cambio, ambos modelos, construccionalización y lexicalización, son imprescindibles.

PALABRAS CLAVE: perífrasis incoativa, verbo de cambio, cuasi-sinonimia, construccionalización, lexicalización. lexical types. In order to explain the processes of change, both models, constructionalization and lexicalization, are indispensable.

KEYWORDS: inchoative periphrasis, change-of-state verb, near-synonymy, constructionalization, lexicalization

\section{INTRODUCCIÓN}

En español, el aspecto incoativo se expresa, entre otros recursos, mediante perífrasis verbales con infinitivo. A lo largo de la historia, varias categorías verbales han suministrado los núcleos auxiliares ${ }^{1}$ de esta construcción, a saber los verbos léxicamente ingresivos (empezar a/comenzar a bailar), los verbos de movimiento (echarse a reír), y los verbos de colocación (ponerse a escribir, meterse a trabajar) (Gómez Manzano 1992; Gómez Torrego 1999; Carrasco Gutiérrez et alii 2006; Aparicio et alii 2014). El presente estudio se concentra en el desarrollo de un cuarto tipo, a saber la construcción incoativa con el verbo de cambio de estado romper como núcleo verbal (romper a llorar).

Romper $a+$ infinitivo es una perífrasis incoativa próxima a echar(se) $a+$ infinitivo o soltarse $a+$ infinitivo. Expresa el inicio de un evento inesperado y altamente dinámico, pero "añade la idea de acción contenida hasta un determinado momento, por lo que se inicia con una mayor brusquedad" (García González 1992: 70; también Fogsgaard 2001; Aparicio et alii 2014). En el ejemplo (1), el público contiene sus emociones hasta que sea aceptable aplaudir.

(1) "Es que tengo a mi madre muy enferma", explicaba María, visiblemente emocionada minutos antes de que los bomberos bajaran la imagen. En cuanto lo consiguieron, el público de la plaza rompió a aplaudir. (CORPES XXI: Alonso C., 2006).

Este significado de inicio brusco retoma en buena medida la semántica básica de romper que se resume como "separar con más o menos violencia las partes de un todo, deshaciendo su unión" (Diccionario de La Lengua Española, en línea). También el aspecto incoativo "tener principio, empezar, comenzar" forma parte de su uso general, como ilustran los casos romper el día, romper a hablar, romper la marcha (Diccionario de La Lengua Española, en línea) y romper el fuego, romper las hostilidades (Diccionario del Uso del Español, Moliner). Según Corominas (1954), también sus derivados nominales rompimiento o ruptura pueden denotar "el comienzo de una acción continuada" como en el rompimiento de la fiesta, de la molienda. Por lo tanto no debe sorprender su funcionamiento en la perífrasis incoativa. Es más, según Väänanen (1968: 149), los antepasados de la construcción que nos ocupa aquí ya se documentan en latín vulgar, bajo la forma erumpere + infinitivo (2).

(2) Erumpunt dicere. (Tertuliano, Adversus Marcionem, I, 17)

1 En este estudio se utiliza el término general auxiliar, siguiendo a Hernanz (1999: 2201) y Gómez Torrego (1999: 3373-3374), aunque otros autores prefieren hablar de semi-auxiliar para distinguir verbos como romper o poner de los auxiliares puros como haber (Borillo 2005). 
Väänänen (1968: 149) propone como traducción ils éclatent en disant. Según el mismo autor, la construcción se expande en latín tardío cuando entra en competición con la variante en que el infinitivo está precedido de la preposición ad (de tipo erumpunt ad dicere). Esta estructura alternante nació, o bien por influencia griega, o bien por contaminación de dos construcciones equivalentes: ad + gerundio, la estructura básica para expresar finalidad en latín clásico (3a), y la variante con infinitivo sin preposición que surgió más tarde, en latín tardío (3b).

(3) a. Aggredior ad dicendum.

b. Aggredior dicere.

Asalto para decir.

Es probable que la contracción de los dos patrones sintácticos haya dado luz a la construcción verbo $+a d+$ infinitivo, la construcción originaria de las perífrasis incoativas en las lenguas romances.

Más que describir en detalle el desarrollo de un patrón sintáctico hasta la fecha poco estudiado, el objetivo de este artículo es reflexionar acerca de las relaciones entre varias teorías del cambio lingüístico en el surgimiento de las perífrasis verbales incoativas españolas. Efectivamente, el proceso mediante el cual los verbos léxicos antedichos - es decir, los verbos ingresivos, de movimiento, colocación o cambio de estado - se han desarrollado como verbos auxiliares ha sido integrado en teorías de la lexicalización (p.ej. Garachana Camarero 2016), gramaticalización (p.ej. Lamiroy y De Mulder 2011), y, más recientemente, construccionalización (Comer y Enghels 2017; Enghels y Comer 2018, en prensa).

La teoría de gramaticalización, sin duda la más difundida, estudia ante todo los procesos de reducción sintáctica y de blanqueamiento semántico sufridos por determinadas expresiones referenciales. Así por ejemplo, Verroens (2011) reconstruye el proceso de gramaticalización del verbo de colocación francés mettre (como en se mettre à travailler, meterse) ponerse a trabajar) proponiendo una evolución en seis fases sucesivas. El momento clave -o mejor dicho, el contexto puente- para entender el surgimiento de la construcción incoativa es el momento en que el sujeto agente del acto de colocación desplaza un objeto o un participante incitándole al mismo tiempo a arrancar una actividad o un evento, expresado por el complemento infinitivo (p.ej. Je mets Jean dans sa chambre à faire ses devoirs, lit. Pongo a Juan en su cuarto a hacer sus deberes). Luego, el complemento locativo se suprime de la estructura argumental del verbo y el significado de colocación del verbo principal se debilita a favor de un significado causativo. Finalmente, la lectura causativa da lugar al uso reflexivo e incoativo del verbo principal (p.ej. Jean se met à faire ses devoirs, Juan se pone a hacer sus deberes).

Recientemente, la validez de este modelo ha sido puesta en tela de juicio por Comer y Enghels (2017) y Enghels y Comer (2018, en prensa). Las autoras argumentan que no permite dar cuenta de la complejidad de la construcción incoativa en español por dos motivos: no considera las variaciones sintácticas a las que la perífrasis ha sido sometida a lo largo de su historia (p.ej. la variación preposicional como en se pusieron a trabajar vs. se metieron en comprar una casa, ver sección 3.1), ni integra la variedad léxica del 
núcleo auxiliar. ¿Hasta qué punto la evolución de la perífrasis con meter, por ejemplo, es comparable a la de su cognado con poner y -relevante para el estudio actual-romper? Según el modelo construccionista, la perífrasis incoativa se define como una construcción o pareja convencionalizada de forma y significado (Goldberg 1995, 2006; Croft 2001), compuesta de cuatro componentes o posiciones: el sujeto principal [ $\left.\mathrm{SN}_{1}\right]$, el auxiliar $\left[\mathrm{V}_{\text {inc }}\right]$, la preposición [PREP] y el infinitivo [INF]. La idea principal es que el significado incoativo se expresa por la construcción en su totalidad y supera el aporte semántico de los constituyentes léxicos individuales. El análisis detallado de la evolución formal y funcional de las perífrasis con poner y meter demuestra que la perífrasis incoativa en español se ha desarrollado como un esquema abstracto que se hizo gradualmente más productivo, de acuerdo con la definición general de la construccionalización. Aumenta su frecuencia absoluta y relativa (en decir, en comparación con otras perífrasis incoativas) y atrae más tipos léxicos diferentes en varios componentes de la construcción. Sin embargo, este modelo ha sido verificado empíricamente para la construcción incoativa con verbos de colocación pero su extensibilidad a las demás perífrasis queda sin verificar. Y, se sabe que perífrasis cuasi-sinónimas ${ }^{2}$ no necesariamente se caracterizan por procesos de cambio idénticos (Garachana Camarero 2011).

Así, siguiendo la Nueva Gramática de la Lengua Española (Real Academia Española/ Asociación de Academias de la Lengua 2009), Garachana Camarero (2016) define la perífrasis creada por romper a + infinitivo (1) - así como echar(se) a + infinitivo - más bien como un caso de lexicalización. Efectivamente, en español contemporáneo, la perífrasis romper $a+$ infinitivo parece restringirse a una serie limitada de verbos que surgen en el componente infinitival, como llorar y reír (Gómez Torrego 1988; Carrasco Gutiérrez et alii 2006), y como tal se aproxima a un esquema fraseológico lexicalizado (Brinton y Traugott 2005). Tal análisis se sitúa del otro lado de la teoría de construccionalización que define la perífrasis incoativa como esquema abstracto que gradualmente atrae a más enunciados concretos (o constructs) diferentes.

A la luz de estos acercamientos diferentes, este estudio tiene como objetivo principal analizar el origen y la evolución formal y funcional de la perífrasis incoativa romper a + infinitivo. Quiere, entre otras cosas, contestar a las preguntas siguientes: ¿Cuándo la perífrasis se documenta por primera vez en los corpus diacrónicos? ¿Su evolución es paralela a la de otras perífrasis cuasi-sinónimas? ¿Hasta qué punto su evolución diacrónica corresponde con un proceso de lexicalización o de construccionalización? En otras palabras, verificamos la validez de los dos modelos teóricamente más alejados y, de momento, dejamos de lado la teoría de gramaticalización. El motivo es evidente: la gramaticalización y construccionalización no son modelos competitivos sino complementarios ya que, como afirman Hilpert (2013), Traugott y Trousdale (2013) y Trousdale (2014), comparten buena parte de sus bases teóricas y metodológicas pero focalizan preguntas diferentes. Mientras que la gramaticalización ha dedicado mayor atención al desarrollo

2 Recurrimos al término cuasi-sinónimo porque las perífrasis estudiadas se caracterizan por un alto grado de similitud semántica sin ser sustituibles en todos los contextos. Esto se debe a la polisemia variable de los elementos léxicos incluidos. Efectivamente, como es demostrado también en este artículo, las perífrasis particulares añaden matices semánticos diferentes (ver por ejemplo Gómez Manzano 1992; Aparicio et alii. 2011, 2014). 
de categorías gramaticales a partir de elementos léxicos individuales, la teoría del cambio de construcciones estudia cómo las configuraciones internas y externas de conjuntos de expresiones cambian.

Las premisas principales de las teorías de la lexicalización y la construccionalización, importantes para este estudio, se resumen como tal ${ }^{3}$.

- En ambos casos, el cambio suele ser gradual, unidireccional y el resultado de un proceso de fusión o coalescencia de entidades lingüísticas individuales (Lehmann 2004; Traugott y Trousdale 2013: 34; ver también sección 3.2).

- La diferencia principal consiste en que el resultado de un proceso de lexicalización es un esquema fraseológico con valor semántico pleno, mientras que la construccionalización lleva a esquemas abstractos o construcciones con significado procedural o gramatical. Como dice Lehmann (2002: 15), la lexicalización implica un desplazamiento (shift) a un inventario léxico por lo cual el resultado debe ser estudiado y adquirido por los hablantes. La construccionalización lleva a esquemas presentes en la mente de los hablantes que se activan una y otra vez.

- Frente a las construcciones, los patrones lexicalizados se caracterizan por una frecuencia de uso y una productividad más baja (ver sección 4).

En concreto, el artículo compara los resultados de varias perífrasis incoativas con el fin de determinar si presentan un proceso evolutivo similar. La investigación se desarrolla en tres fases. La sección 2 proporciona más detalles sobre el banco de datos usado y expone los parámetros que definen la variación formal y funcional de las perífrasis incoativas. También resume el desarrollo histórico y las frecuencias de uso generales. Luego, la sección 3 se dedica a la descripción de la evolución morfosintáctica de romper a + infinitivo en comparación con la de ponerse/meterse a + infinitivo. Más concretamente, analiza su fijación formal y la incorporación sintáctica del verbo romper con su infinitivo. La sección 4 se detiene en la productividad de la construcción y en el estudio de la evolución semántico-léxica del infinitivo. La discusión final (sección 5) resume las conclusiones principales y sopesa la aplicabilidad de las teorías de la lexicalización y la construccionalización.

\section{CORPUS Y METODOLOGÍA}

\subsection{Colección de datos}

Para alcanzar los objetivos antedichos, el estudio diacrónico se basa en un amplio corpus compilado y anotado manualmente. Todos los casos de romper a infinitivo han sido extraídos de los bancos de datos Corpus Diacrónico del Español (CORDE) para los siglos XIII hasta XIX, Corpus de Referencia del Español Actual (CREA) para el siglo XX y Corpus del Español del Siglo XXI (CORPES XXI) para el siglo XXI, limitando la búsqueda al español peninsular. Estos datos permiten trazar el desarrollo sintáctico y semántico de la

3 Este estudio no tiene como objetivo ofrecer el estado de la cuestión de las delimitaciones de ambos modelos, sino que, con vistas a los resultados del análisis, se basa en una selección de lecturas relevantes como Himmelmann (2004), Brinton y Traugott (2005), Garachana Camarero (2011), Lehmann (2002, 2004), Traugott y Trousdale (2013) entre otras. 
perífrasis incoativa con romper, $\mathrm{y}$, además, compararlo sistemáticamente con los resultados del análisis paralelo de las perífrasis con ponerse y meterse tal como han sido expuestos en Comer y Enghels (2017) y Enghels y Comer (2018, en prensa).

Se comprueba una primera diferencia notable entre las perífrasis cuasi-sinónimas en la datación de sus primeras atestaciones. Mientras que las variantes con meter (4a) y poner (4b) se documentan ya en textos del siglo XIII, la construcción con romper surge por primera vez en un texto del siglo XIX (5a). En la mayoría de los casos anteriores al siglo $\mathrm{XIX}$, el verbo romper entra en una estructura transitiva de tipo romper $+(a)+S N(5 \mathrm{~b})$ o ditransitiva romper $+($ a) $S N+S N(5 \mathrm{c})$.

(4) a. Et non fallé ninguna escusaçión por que non deviese fincar en la ley del padre. Et quíseme dexar de todo et meterme a fazer pesquisas de las leyes et estudiar en ellas. (CORDE: Anónimo, 1251)

b. Et si se desuariaren; mengua lo menor de lo mayor. \& lo que fincare. toma su sexma parte. \& esso sera ell argumento de una hora del desuariamiento. \& multiplicalo en las horas dell arredramiento del grado delantrero. de qual angulo quier que tu pongas a obrar con ell. (CORDE: Alfonso X, 1276-1277)

(5) a. Comenzó la condesa a dar indicios de querer desahogar su pecho oprimido, y de allí a poco rompió de nuevo a llorar amargas y copiosas lágrimas. (CORDE: de Larra M.J., 1834) b. Ninguna rompe más flecos de oro, ni destroza más encajes, ni pisa mejores piedras. (CORDE: de Torres Villarroel D., 1727-1728)

c. [...] y parecíale ser mal hecho y peor pensado ponerse en peligro de romper a su señora la fee prometida, y decíase a sí mismo: [...] (CORDE: de Cervantes M., 1615)

Sin embargo, como muestra el cuadro 1, a partir del siglo XIX la frecuencia absoluta de la perífrasis incoativa con romper no deja de aumentar. El cuadro 1 también reúne las frecuencias relativas de la perífrasis (es decir por cada 10.000 ocurrencias del verbo romper en los bancos de datos consultados) que apuntan a la misma tendencia creciente.

\begin{tabular}{ccc}
\hline siglo & frec. absoluta & $\begin{array}{c}\text { frec. relativa } \\
(/ 10.000 \text { ocurr. romper })\end{array}$ \\
\hline XIX & 178 & 383 \\
XX & 222 & 378 \\
XXI & 456 & 434 \\
\hline TOTAL & 856 & - \\
\hline
\end{tabular}

Cuadro 1. Frecuencias absolutas y relativas de romper $a+$ infinitivo

Si comparamos estos datos con el desarrollo de las perífrasis con los verbos de colocación ponerse/meterse (cuadro 2), se puede concluir que, a pesar de la diferencia cuantitativa en números absolutos, siendo estas mucho más frecuentes en el discurso, la variante con romper se destaca por su alta frecuencia relativa. En otras palabras, cuando el hispanohablante quiere expresar el valor incoativo, recurre más frecuentemente a los verbos ponerse y meterse, pero asocia el uso de romper más estrechamente a su valor incoativo. 


\begin{tabular}{ccc}
\hline siglo & frec. absoluta & $\begin{array}{c}\text { frec. relativa } \\
(/ 10.000 \text { ocurr. })\end{array}$ \\
\hline XIII & 50 & 46 \\
XV & 148 & 46 \\
XVII & 734 & 114 \\
XIX & 1210 & 273 \\
XXI & 3001 & 287 \\
\hline TOTAL & 5143 & -
\end{tabular}

Cuadro 2. Frecuencias absolutas y relativas del uso ponerse/meterse $a+$ infinitivo $^{4}$

\subsection{Parámetros de análisis}

A fin de analizar el proceso evolutivo de romper $a+$ infinitivo analizamos tres parámetros que se consideran como diagnósticas fiables del cambio lingüístico (entre otros Hilpert 2013), tanto en términos de construccionalización como lexicalización, y de auxiliarización. Más en concreto se trata de detectar cambios pertinentes en cuanto a su forma y semántica y observar posibles evoluciones en la distribución de variantes estructurales y/o funcionales. Esta variación, que se describe a continuación en las secciones 3 y 4, se relaciona con los parámetros siguientes:

1. el tipo de preposición que introduce el complemento infinitivo subordinado y que mide el grado de fijación formal de la perífrasis (sección 3.1);

2. la posibilidad o no de intercalar elementos léxicos, y en particular complementos adverbiales, entre el verbo conjugado y el infinitivo, lo cual apunta a respectivamente un menor o mayor grado de incorporación de ambos verbos (sección 3.2);

3. los rasgos léxico-semánticos de los constituyentes principales de la construcción, a saber el sujeto-agente principal y el infinitivo subordinado. Este criterio permite estudiar en qué medida la perífrasis se ha desarrollado como construcción o esquema más abstracto, incluyendo tipos léxico-semántico menos prototípicos (sección 4).

\section{DESARROLLO MORFOSINTÁCTICO DE LA PERÍFRASIS ROMPER $A+$ IN- FINITIVO}

Dos criterios permiten examinar en qué medida la construcción incoativa da muestras de una gradual fijación morfosintáctica, a saber la preposición que se utiliza para encabezar el complemento infinitivo subordinado (sección 3.1), y el grado de incorporación entre el verbo auxiliar y el infinitivo (sección 3.2).

4 Nótese que por la gran cantidad de ejemplos, el corpus con poner y meter se ha compuesto a partir de cinco cortes cronológicos o intervalos regulares. Para más informaciones sobre las ventajas de aplicar este método, ver Enghels y Comer (2018). 


\subsection{Fijación formal: $V+a+$ Inf vs. $V+e n+$ Inf}

En su estudio sobre poner y meter, Comer y Enghels (2017) y Enghels y Comer (2018, en prensa) observan que, en épocas anteriores, la perífrasis incoativa se construyó no solo con la preposición $a(6 \mathrm{a})$, sino también con en (6b), e incluso sin preposición (6c).

(6) a. [...] que osadía ha sido poner a hacer esto delante de quien tantas voces mejores que la mía habría oído. (CORDE: de Castillo Solórzano A., 1642)

b. [...] no se meta en escreuir letras amorosas ni plazenteras [...]. (CORDE: anónimo, 1448) c. [...] y no menos Jeremías se ponía pregonar la venida del mexías [...]. (CORDE: del Encina, $1481-1496)^{5}$

La variabilidad sintáctica se observa ante todo entre los siglos XIII y XV. En una fase posterior, a partir del siglo XVII, tiene lugar una fijación formal de la perífrasis que consiste en la extinción de las variantes con en y sin preposición y el uso exclusivo de la preposición a. La aparición de la variante con en ha sido invocada como la clave para entender el desarrollo del esquema incoativo. Comer y Enghels (2017) argumentan que la perífrasis se ha formado mediante una extensión metafórica del esquema locativo de movimiento causado (caused-motion, p.ej. Goldberg 1995:52). Según este esquema, una causa hace que un tema se desplace a otro lugar, la meta, expresada mediante un complemento oblicuo generalmente introducido por la preposición en en español (poner algo en un sitio). La idea es que a partir de este esquema básico, otro subesquema ha surgido en que un participante pone a otro participante en un lugar abstracto, siendo el evento subordinado expresado por el infinitivo e introducido por la preposición en (poner algo/alguien en un evento). En la variante con la preposición $a$, el hablante conceptualiza el evento (infinitivo) subordinado más bien como un objetivo por alcanzar en un futuro inmediato. Después de una fase de competición entre las variantes formales, la construcción con $a$ se hace dominante porque resulta más compatible con el significado de finalidad de la perífrasis incoativa.

Ahora bien, como la perífrasis incoativa con romper no aparece en los datos antes del siglo XIX, es decir, después de la fase de fijación preposicional de la construcción, era de esperar que la variación no se documentara, lo cual se confirma por el corpus.

\subsection{Fijación formal: el grado de incorporación sintáctica}

El segundo parámetro formal se relaciona con el orden de palabras y examina hasta qué punto es posible intercalar un elemento léxico, como un adverbio, entre el auxiliar y el infinitivo. Observamos la siguiente variación: o bien la perífrasis coincide con la estructura [SN $+\mathrm{AUX}+(\mathrm{PREP}+\mathrm{INF})+\mathrm{ADV}]$, o bien con $[\mathrm{SN}+\mathrm{AUX}+\mathrm{ADV}+(\mathrm{PREP}+\mathrm{INF})]$. Esta variación indica el grado de incorporación y unidad sintáctica de la construcción incoativa. Cuanto más la construcción se fija, más el verbo auxiliar y el infinitivo subordinado forman un predicado complejo que no admite intercalación de elementos léxicos (Fauconnier 1983;

5 Un lector atento nos sugirió que la ausencia de preposición podría explicarse por motivos fonéticos, y más particularmente la vocal final - $a$ de ponía. Para evitar el encadenamiento de dos veces la misma vocal, la preposición se suprime. Sin embargo, se observan varios casos de ausencia de preposición en el corpus con formas verbales que acaban en otra vocal (p.ej. muy despacio me puse considerar las desdichas de algunos hombres). 
Fischer 2007: 193; Enghels 2007: 150). Por lo tanto, menos variación estructural habrá. En el ejemplo (7a) no se intercala ningún elemento léxico entre romper y el infinitivo (excepto, por supuesto, la preposición a) y el adverbio también se pospone, contrariamente a la estructura con intercalación del adverbio también en (7b).

(7) a. La Niña ríe, alegre por su ocurrencia. La Abuela, al ver reír a La Niña, rompe a reír también. (CREA: Olmo L., 1963)

b. Que me haga usted el favor de dar la cuenta, dice la cocinera, rompiendo también $a$ llorar, y arrojando el mandil sobre una silla, como rey que depone su corona. (CORDE: de Pereda J.M., 1878)

Enghels \& Comer (en prensa) observan una tendencia continua y creciente hacia el uso de la variante sin intercalación de elementos léxicos, lo cual apunta a una reducción de la variabilidad formal en la perífrasis con poner y meter. Los datos, retomados en el cuadro 3, soportan la hipótesis según la cual la perífrasis incoativa se ha desarrollado como una construcción o un esquema más fijo.

\begin{tabular}{ccccccc}
\hline \multirow{2}{*}{ siglo } & \multicolumn{2}{c}{+ intercal } & \multicolumn{2}{c}{ - intercal } & \multicolumn{2}{c}{ Total } \\
\cline { 2 - 7 } & $\#$ & $\%$ & $\#$ & $\%$ & $\#$ & $\%$ \\
\hline XIII & 16 & 32 & 34 & 68 & 50 & 100 \\
XV & 19 & 12.8 & 129 & 87.2 & 148 & 100 \\
XVII & 92 & 12.5 & 642 & 87.5 & 734 & 100 \\
XIX & 117 & 9.7 & 1093 & 90.3 & 1210 & 100 \\
XXI & 137 & 4.6 & 2864 & 95.4 & 3001 & 100 \\
\hline
\end{tabular}

Cuadro 3. Intercalación elementos léxicos entre ponerse/meterse e infinitivo

Una vez más, como la variante con romper surge en un momento cuando el esquema parece haberse establecido ya, esperamos observar poca variabilidad sintáctica.

Efectivamente, el corpus contiene en total solo 13 casos (sobre 856) de un adverbio intercalado entre el auxiliar romper y el infinitivo. Además, los elementos intercalados se restringen a dos tipos léxicos, a saber los adverbios también (10 casos, cf. ejemplo (7b)) y de nuevo (cf. supra ejemplo 5a). Recogemos 4 ejemplos en el corpus del siglo XIX, 8 en el siglo XX y un solo ejemplo en los datos del siglo XXI (8).

(8) [...] la infanta Cristina y su esposo, Iñaki de Urdangarín, rompieron también a llorar y tuvieron que ser consolados por los príncipes de Jordania [...]. (CORPES XXI: Abejón P., 2004)

Los datos muestran que la variación sintáctica ya no se presenta en español actual, y romper $a+$ infinitivo se comporta como estructura altamente incorporada.

Del análisis que precede se puede concluir que, dado que el verbo de cambio de estado romper ha sido atraído por la construcción incoativa en un momento en que el esquema incoativo ya se había formado (con la preposición $a$ que introduce el complemento infinitivo y la fuerte unión entre este y el V auxiliar), se observa poca variación sintáctica. De este 
modo, los datos parecen confirman la hipótesis de construccionalización de la perífrasis incoativa en español: una vez anclado el esquema en la mente de los hablantes, atrae a más elementos léxicos (en el caso concreto, tras poner y meter, el verbo romper) que no influyen en la forma del esquema. La sección 4 examina hasta qué punto el desarrollo léxicosemántico de la perífrasis apunta a la misma conclusión.

\section{EVOLUCIÓN FUNCIONAL DE LA PERÍFRASIS ROMPER $A$ + INFINITIVO}

\subsection{Formulación de hipótesis}

Más que los rasgos formales, las características semánticas permiten verificar a qué modelo de cambio lingüístico el desarrollo de la perífrasis romper $a+$ infinitivo se ajusta. Dos puntos de vista se oponen. Por un lado, los fenómenos de construccionalización suelen coincidir con mayor productividad léxico-semántica. Cuando una estructura se desarrolla como esquema, los hablantes hacen abstracción de los tipos semánticos originariamente incorporados e incluyen nuevos tipos, menos prototípicos (cf. el fenómeno de host-class expansion de Himmelmann 2004; Barðdal 2008; Hilpert 2013; Traugott y Trousdale 2013 entre otros). Por el otro lado, los fenómenos de lexicalización implican recurrencias léxicas en los componentes de una construcción, por lo cual el esquema está lejos de ser productivo (Brinton y Traugott 2005; Garachana Camarero 2016 entre otros muchos).

García González (1992) sostiene que el uso de la perífrasis romper + infinitivo es poco frecuente y se limita a registros cultos salvo con los infinitivos reír y llorar, afirmación que va en dirección de una fuerte lexicalización de la perífrasis. Además, ya sabemos que (cf. sección 1), frente a otras perífrasis incoativas, romper $a+$ infinitivo añade el matiz de que el evento se inicia brusca e inesperadamente. Por consiguiente, es de esperar que el infinitivo denote exclusivamente eventos dinámicos (*romper a ser, *romper a amar/odiar etc.). A fin de obtener una visión completa de su desarrollo funcional, descomponemos el análisis semántico en dos fenómenos interrelacionados. Investigamos la evolución de los tipos semánticos que denota la construcción entera, dedicando atención particular al aspecto léxico (es decir ¿el evento iniciado solo refiere a acciones dinámicas o se extiende también a otros tipos aspectuales?) (sección 4.2), pero también la clase léxica del infinitivo mismo (sección 4.3).

\subsection{Esquemas semánticos generales y aspectuales}

Teniendo en cuenta la animacidad del sujeto principal (SN), y la dinamicidad del infinitivo (que puede expresar sí o no una transferencia de energía), Enghels y Comer (2018) demuestran que la perífrasis incoativa puede responder a seis esquemas semánticos diferentes:

- tipo 1: SN [+ANIMADO] + INF [+DINÁMICO] (ej. el padre se pone a leer)

- tipo 2: SN [+ANIMADO] + INF [-DINÁMICO] (ej. cuando me pongo a ser mala, lo soy de veras)

- tipo 3: SN [-ANIMADO] + INF [+DINÁMICO] (ej. su labio se puso a temblar)

- tipo 4: SN [-ANIMADO] + INF [-DINÁMICO] (ej. su dedo se puso a sangrar)

- tipo 5: [IMPERSONAL] + INF [+DINÁMICO] (ej. se puso a llover)

- tipo 6: [IMPERSONAL] + INF [-DINÁMICO] 
El cuadro 4 retoma sus resultados y muestra la evolución de la frecuencia relativa de los seis tipos semánticos por corte cronológico destacado.

\begin{tabular}{ccccccccccccccccc}
\hline & \multicolumn{1}{c}{1} & \multicolumn{1}{c}{2} & \multicolumn{1}{c}{3} & \multicolumn{3}{c}{4} & \multicolumn{3}{c}{5} & \multicolumn{3}{c}{6} & \multicolumn{3}{c}{ total } \\
\cline { 2 - 15 } & $\#$ & $\%$ & $\#$ & $\%$ & $\#$ & $\%$ & $\#$ & $\%$ & $\#$ & $\%$ & $\#$ & $\%$ & $\#$ & $\%$ \\
\hline XIII & 48 & 96 & 2 & 4 & - & - & - & - & - & - & - & - & 50 & 100 \\
XV & 140 & 94.6 & 4 & 2.7 & 4 & 2.7 & - & - & - & - & - & - & 148 & 100 \\
XVII & 702 & 95.6 & 19 & 2.6 & 12 & 1.6 & 1 & 0.2 & - & - & - & - & 734 & 100 \\
XIX & 1163 & 96.1 & 20 & 1.7 & 23 & 1.9 & 4 & 0.3 & - & - & - & - & 1210 & 100 \\
XXI & 2848 & 94.9 & 21 & 0.7 & 86 & 2.9 & 27 & 0.9 & 2 & 0.1 & 17 & 0.6 & 3001 & 100 \\
\hline
\end{tabular}

Cuadro 4. Evolución tipos semánticos ponerse/meterse a infinitivo

Los datos muestran que en el siglo XIII, la construcción incoativa con los verbos de colocación se restringió casi exclusivamente al tipo semántico prototípico SN [+ANIMADO] + INF [+DINÁMICO] (96\%). Este esquema sigue siendo dominante en el español contemporáneo aunque la construcción incluye gradualmente (pero siempre de manera limitada) más tipos semánticos diversos, con sujetos inanimados, incluso esquemas impersonales e infinitivos no dinámicos. Por tanto, es lícito concluir que la perífrasis con poner/meter se ha hecho semánticamente más productiva, lo cual coincide con su construccionalización.

Comparemos estos datos con los de romper $a+$ infinitivo, recogidos en el cuadro 5.

\begin{tabular}{cccccccccccccccccc}
\hline & \multicolumn{2}{c}{1} & \multicolumn{1}{c}{2} & \multicolumn{1}{c}{3} & & \multicolumn{1}{c}{4} & \multicolumn{1}{c}{5} & \multicolumn{2}{c}{6} & \multicolumn{2}{c}{ total } \\
\cline { 2 - 15 } & $\#$ & $\%$ & $\#$ & $\%$ & $\#$ & $\%$ & $\#$ & $\%$ & $\#$ & $\%$ & $\#$ & $\%$ & $\#$ & $\%$ \\
\hline XIX & 170 & 95.5 & - & - & 8 & 4.5 & - & - & - & - & - & - & 178 & 100 \\
XX & 203 & 91.4 & - & - & 18 & 8.1 & - & - & 1 & 0.5 & - & - & 222 & 100 \\
XXI & 339 & 74.3 & - & - & 111 & 24.3 & - & - & 6 & 1.4 & - & - & 456 & 100 \\
\hline
\end{tabular}

Cuadro 5. Evolución tipos semánticos romper a + infinitivo

De la tabla resalta que el alcance semántico de la perífrasis con romper es mucho más restringido que sus cognados con poner y meter. Otra vez predomina el esquema prototípico SN [+ANIMADO] + INF [+DINÁMICO] (tipo 1) (9). De acuerdo con el comportamiento esperado, la perífrasis no selecciona infinitivos no dinámicos.

(9) a. Aquí rompió a llorar la hermana de Tolín, como si el alma se le saliera por la boca. (CORDE: de Pereda J.M., 1885,)

b. Como los mayores rompieron a reír, Miguelí se figuró que la escena debió resultar bastante cómica. (CREA: Rivarola Matto, J.B., 1970)

Sin embargo, es interesante constatar que, contrariamente a las perífrasis con poner $y$ meter, la variante con romper incorpora más frecuentemente sujetos inanimados, y eso en 
tendencia alcista. Efectivamente, la frecuencia relativa del tipo 3 SN [-ANIMADO] + INF [+DINÁMICO] sube del 8.6\% en el siglo XIX al 24.3\% en el corpus del siglo XXI (10a-b). También ocurre más regularmente el esquema tipo 5 [IMPERSONAL] + INF [+DINÁMI$\mathrm{CO}]$ que se limita al solo verbo llover (10c).

(10) a. Se pone la cacerola sobre fuego vivo para que rompa a cocer, y en seguida se tapa y se sigue la cocción a fuego lento durante tres horas. (CORDE: Muro, A., 1891)

b. Al día siguiente los ponemos a cocer con agua a fuego lento. Cuando rompa a hervir, subimos el fuego y los dejamos cocer un par de minutos. (CORPES XXI: Sanjuán G., 2004) c. Afuera rompió a llover con fuerza. (CORPES XXI: Abella R., 2009)

No obstante, a fin de tener una visión más clara del rasgo de dinamicidad del infinitivo es necesario analizar algunos criterios aspectuales adicionales.

Aparicio et alii (2014) señalan que inicialmente las construcciones incoativas solo seleccionan eventos dinámicos y télicos y que solo más tarde incluyen también infinitivos no dinámicos y atélicos. Distinguen seis clases aspectuales, a saber los estados, procesos, puntos, culminaciones, realizaciones y eventos graduales que se definen en el cuadro 6 .

\begin{tabular}{lccccc}
\hline & dinamicidad & telicidad & duración & gradualidad & ejemplos \\
\hline estado & - & - & + & - & equivaler, ser, conocer \\
proceso & + & - & + & - & caminar, buscar, llorar, reir \\
punto & + & - & - & - & pestañear, saltar, toser \\
culminación $^{1}$ & + & + & - & - & capturar, llegar, cerrar \\
realización & + & + & + & - & construir, aprender, instalar \\
gradual $^{2}$ & + & + & + & + & envejecer, engordar, calentar \\
\hline
\end{tabular}

Cuadro 6. Síntesis de rasgos y clases aspectuales (Aparicio et al. 2011: 25)

Aparicio et alii (2014: 86) sostienen que la construcción incoativa con romper solo se construye con infinitivos que denotan procesos (dinámicos y durativos) o puntos (dinámicos y puntuales). Ahora bien, si clasificamos todos los casos del corpus, obtenemos los datos del cuadro 7.

\begin{tabular}{ccccccccccccccc}
\hline & \multicolumn{2}{c}{ estado } & \multicolumn{2}{c}{ proceso } & \multicolumn{2}{c}{ punto } & \multicolumn{2}{c}{ culminación } & \multicolumn{2}{c}{ realización } & \multicolumn{2}{c}{ gradual } & \multicolumn{2}{c}{ total } \\
\cline { 2 - 17 } & $\#$ & $\%$ & $\#$ & $\%$ & $\#$ & $\%$ & $\#$ & $\%$ & $\#$ & $\%$ & $\#$ & $\%$ & $\#$ & $\%$ \\
\hline XIX & - & - & 174 & 97.8 & 3 & 0.5 & - & - & 1 & 0.6 & - & - & 178 & 100 \\
XX & - & - & 211 & 95 & 6 & 2.7 & - & - & 5 & 2.3 & - & - & 222 & 100 \\
XXI & - & - & 449 & 98.5 & 4 & 0.9 & - & - & 3 & 0.7 & - & - & 456 & 100 \\
\hline
\end{tabular}

Cuadro 7. Evolución clases aspectuales romper a infinitivo

6 Una culminación es un evento complejo formado por un punto y una consecuencia, generalmente un estado; coincide con la clase de logro de Vendler (1967).

7 Los eventos graduales son formados por una iteración de culminaciones, se parecen mucho a las realizaciones pero al contrario de estas, el avance del evento es gradual. 
Del análisis resulta claro que la clase aspectual de los procesos predomina en todos los siglos, y que otras clases como los puntos y las realizaciones solo aparecen de manera excepcional en el corpus. La dominancia de la clase aspectual de los procesos se explica por la abundancia de los infinitivos llorar y reír, lo cual parece confirmar la idea de una fuerte lexicalización de la construcción. Los infinitivos que denotan procesos más frecuentes son, en orden decreciente: llorar (329/834) (11a), reír $(\mathrm{n}=148)(11 \mathrm{~b})$, hablar $(\mathrm{n}=78)(11 \mathrm{c})$, cantar $(\mathrm{n}=38)(11 \mathrm{~d})$, andar $(\mathrm{n}=16)(11 \mathrm{e})$, aplaudir $(\mathrm{n}=12)(11 \mathrm{f})$, ladrar $(\mathrm{n}=8)(11 \mathrm{~g})$, correr $(\mathrm{n}=7)(11 \mathrm{~h})$.

(11) a. Aquí rompió a llorar la hermana de Tolín, como si el alma se le saliera por la boca. (CORDE: de Pereda J.M., 1885)

b. Todos rompieron a reír, mientras él daba paso a un trago de vino. (CORDE: Pérez Galdós B., 1876)

c. ¡Ay!, los mudos que rompen a hablar son terribles. (CORDE: Picón Jacinto O., 1884)

d. Con que ya no pude resistir más, y rompí a cantar, [...]. (CORDE: Echagaray J., 1895)

e. Martín Marco se sujeta sus gafas de cerquillo de alambre y rompe a andar. (CREA: Camilo Cela J.,1951)

f. [...] en cuanto asomamos las narices, rompieron a aplaudir [...]. (CORDE: Zorrilla J., 1883)

g. Cuando me sintieron los perros, rompieron a ladrar [...]. (CORDE: Echagaray J., 1895)

h. [...] saltó a la vía y rompió a correr hacia la fonda. (CORDE: Pardo Bazán E., 1881)

Los verbos que denotan puntos son: disparar (12a), embestir (12b) y toser (12c).

(12) a. El caballo que, asustándose, rompe a disparar, [...]. (CORDE: Baros A., 1875)

b. El toro además tuvo a su favor que respondía y repetía, que una vez que rompía a embestir no paraba. (CORPES XXI: Zabala de la Serna V., 2012)

c. Diciendo esto, rompió a toser con tanta fuerza, que parecía que se le desgarraba el pecho y que iba a arrojar las entrañas por la boca. (CORDE: Pérez Galdós, B., 1883)

Finalmente, el corpus contiene 9 atestaciones de infinitivos que denotan realizaciones, entre los que predominan leer (13a) y tocar (13b).

(13) a. Ya se iba a dar tierra al cadáver, cuando, al lado del mismo, se adelantó un joven de menudo cuerpo, larga y negra cabellera, mal enlutado con ropas ajenas, el cual rompió a leer una composición [...]. (CORDE: Menéndez Pidal R., 1910)

b. Los músicos rompieron a tocar un vals juguetón y alegre, colocándose detrás del féretro [...]. (CORDE: Blasco Ibañez V., 1898)

Antes de terminar este apartado, es interesante comparar brevemente estos resultados con los datos que nos prevén Aparicio et alii (2014: 82) de las clases aspectuales seleccionadas por la construcción incoativa con ponerse.

\begin{tabular}{cccccc}
\hline procesos & realizaciones & puntos & graduales & culminaciones & estados \\
\hline $58 \%$ & $15 \%$ & $14 \%$ & $11 \%$ & $2 \%$ & $0.001 \%$ \\
\hline
\end{tabular}

Cuadro 8. Clases aspectuales ponerse $a+$ infinitivo 
Resulta que en su corpus la construcción incoativa con ponerse demuestra más variabilidad aspectual, dado que las 6 clases están presentes, incluso eventos graduales (p.ej. se puso a alisar su desordenada cabellera), culminaciones (p.ej. me puse a encender las luces) y un caso con un verbo de estado (p.ej. se puso a amar lo pequeño del mundo). Una vez más los datos apuntan a que la construcción incoativa con ponerse sea más productiva que la variante con romper que parece más lexicalizada. Para consolidar esta afirmación pasamos al análisis léxico del infinitivo subordinado.

\subsection{Análisis léxico del complemento infinitivo}

La segunda parte del análisis de la productividad de la perífrasis incoativa se basa en la idea de que cuanto más se ha desarrollado como esquema abstracto, más tipos léxicos diferentes pueden llenar un componente vacío de la construcción (Himmelmann 2004; Bybee y Cacoullos 2009: 189). Por consiguiente, anotamos todos los tipos léxicos que aparecen en la posición del infinitivo en el corpus. Luego determinamos la medida de productividad de la perífrasis incoativa en cada siglo estudiado calculando el type/token ratio, a saber la proporción de los tipos léxicos diferentes (type) teniendo en cuenta el número de ocurrencias en cada subcorpus (tokens). El cuadro 9 compara las medidas de productividad de las perífrasis con ponerse/meterse con las de la variante con romper. Resulta que, en general, la productividad de las incoativas con verbos de colocación es más alta que la de la construcción con romper, aunque la diferencia se hace menos acusada en el subcorpus más reciente. Se podría concluir que en la fase anterior de la formación del esquema incoativo (siglos XIII-XVII) la construcción gradualmente se abrió a incluir más tipos léxicos diferentes. Sin embargo, en cuanto se haya formado el esquema abstracto, las perífrasis con ponerse y meterse entran gradualmente más en competición con estructuras cuasi-sinónimas que tienen otras clases verbales como auxiliar. Es posible que a partir de este momento, una parte del campo semántico se especialice y que determinados infinitivos lleguen a combinarse más con determinados verbos auxiliares (en el caso concreto, romper). Esta hipótesis queda sin verificar para otros tipos de perífrasis con echarse, empezar, comenzar, etc.

\begin{tabular}{ccc}
\hline & incoativo $_{\text {colocación }}$ & incoativo $_{\text {romper }}$ \\
\hline XIII & $0.6(32 / 50)$ & - \\
XV & $0.7(99 / 148)$ & - \\
XVII & $0.4(259 / 734)$ & - \\
XIX & $0.3(395 / 1210)$ & $0.08(15 / 178)$ \\
XX & - & $0.10(23 / 222)$ \\
XXI & $0.2(555 / 3301)$ & $0.06(28 / 456)$ \\
\hline
\end{tabular}

Cuadro 9. Comparación type/token ratio 
Sea cual sea, los datos sugieren que la perífrasis con romper ni con mucho se restringe a los verbos llorar y reír. En total, se distinguen 36 tipos léxicos diferentes que se acumulan a través de los tres siglos estudiados. De hecho, en cada siglo se observa una serie de nuevos tipos léxicos o hápax que también apuntan a cierta productividad y que permiten calcular la productividad potencial (Hilpert 2013: 128) de la construcción, es decir el número de hapaxes por ocurrencias en el subcorpus. En el siglo XX la medida asciende a $0.05(12 / 222)$, en el siglo XXI baja a 0.02 (8/456). Entonces, aunque de manera limitada, también la perífrasis con romper es productiva en español contemporáneo.

Como último paso, nos proponemos investigar si la evolución semántica atañe a determinados campos. Para este propósito nos sirve la clasificación de ADESSE (Alternancias de Diátesis y Esquemas Sintáctico-Semánticos del Español, Albertuz 2007) que distingue seis categorías léxicas principales:

- tipo 1: verbos mentales - sensación (gustar), percepción (ver), cognición (entender)

- tipo 2: verbos materiales - espacio (mover), cambio (romper), comportamiento (crear), meteorología (llover)

- tipo 3: verbos verbales - comunicación (hablar), valorización (criticar), emisión de ruido (ladrar)

- tipo 4: verbos relacionales - atribución (ser), posesión (tener)

- tipo 5: verbos de modulación - causación (hacer), disposición (permitir), verbos soporte (dar)

- tipo 6: verbos existenciales - existencia (ocurrir), fase tiempo (empezar), vida (nacer)

Las incoativas con ponerse/meterse seleccionan estas clases léxicas según las frecuencias presentadas en el cuadro 10 (Enghels y Comer en prensa).

\begin{tabular}{cccccccccccccccc}
\hline & \multicolumn{1}{c}{1} & \multicolumn{1}{c}{2} & \multicolumn{2}{c}{3} & \multicolumn{2}{c}{4} & \multicolumn{3}{c}{5} & \multicolumn{3}{c}{6} & \multicolumn{2}{c}{ total } \\
\cline { 2 - 15 } & $\#$ & $\%$ & $\#$ & $\%$ & $\#$ & $\%$ & $\#$ & $\%$ & $\#$ & $\%$ & $\#$ & $\%$ & $\#$ & $\%$ \\
\hline XIII & 6 & 12 & 25 & 50 & 7 & 14 & 4 & 8 & 8 & 16 & - & - & 50 & 100 \\
XV & 29 & 21.1 & 54 & 39.1 & 15 & 10.9 & 17 & 12.3 & 17 & 12.3 & 6 & 4.3 & 138 & 100 \\
XVII & 232 & 31.6 & 237 & 32.3 & 140 & 19.1 & 43 & 5.9 & 36 & 4.9 & 18 & 2.5 & 734 & 100 \\
XIX & 317 & 30.7 & 458 & 44.3 & 274 & 26.5 & 30 & 2.9 & 20 & 1.9 & 19 & 1.8 & 1033 & 100 \\
XXI & 626 & 22.2 & 1499 & 53.2 & 561 & 19.9 & 46 & 0.6 & 63 & 2.2 & 22 & 0.8 & 2817 & 100 \\
\hline
\end{tabular}

Cuadro 10. Evolución clases léxicas ponerse/meterse $a+$ infinitivo $^{8}$

Una vez más se confirma la alta productividad semántica de la construcción. Ya a partir del siglo $\mathrm{XV}$, los seis tipos destacados se observan en el corpus. Los campos léxicos pre-

8 Nótese que algunos casos son ambiguos y no se dejan clasificar en una sola categoría. Por ejemplo, en se pone a escribir una carta al rey, el infinitivo escribir se clasifica tanto como verbo de creación del tipo 2 que como verbo de comunicación del tipo 3. Estos ejemplos no están incluidos en la tabla. 
feridos siempre han sido los verbos materiales (53.2\%) (14a), mentales (22.2\%) (14b) y verbales (19.9\%) (14c). Aun así, se observa una baja considerable de los verbos relacionales (del $8 \%$ en el siglo XIII hasta el $0.6 \%$ en el siglo XXI) (14d) y de modulación (del 16\% en el siglo XIII hasta el $2.2 \%$ en el siglo XXI) (14e).

(14) a. Recordé que también guardaba seis botellas intactas de Sangre y Trabajadero, que me había enviado un antiguo y amable amigo de Cádiz, pero me daba pereza ponerme a abrir a aquellas horas una caja claveteada. (CORPES XXI: Marías J., 2007)

b. E como cada vno se metio a aprender çiençia \& letras. (CORDE: Mejía F., 1477-1485) c. [...] se puso a contarles un cuento de antes de dormir. (CORPES XXI: Mestre J., 2011) d. Si alguien se pone a cobrar el coste de una representación, de inmediato nos damos cuenta de que no hay mercado que lo resista, [...] (CORPES XXI, anónimo, 2001)

e. Al estampido de los tiros, acudieron los vencedores milicianos y soldados del ejército, y se pusieron á dar alcance á los malaventurados Guardias reales [...]. (CORDE: Alcalá Galiano A., 1847-1849)

La imagen que resalta en el cuadro 11 es muy diferente, y confirma la menor productividad léxica de la construcción con romper.

\begin{tabular}{ccccccccccccccccc}
\hline & \multicolumn{3}{c}{1} & \multicolumn{2}{c}{2} & \multicolumn{2}{c}{3} & \multicolumn{2}{c}{4} & \multicolumn{2}{c}{5} & \multicolumn{3}{c}{6} & \multicolumn{2}{c}{ total } \\
\cline { 2 - 14 } & $\#$ & $\%$ & $\#$ & $\%$ & $\#$ & $\%$ & $\#$ & $\%$ & $\#$ & $\%$ & $\#$ & $\%$ & $\#$ & $\%$ \\
\hline XIX & - & - & 125 & 70.2 & 53 & 29.8 & - & - & - & - & - & - & 178 & 100 \\
XX & 1 & 0.5 & 156 & 69.8 & 64 & 28.8 & 1 & 0.9 & - & - & - & - & 222 & 100 \\
XXI & 1 & 0.2 & 390 & 85.5 & 65 & 14.2 & - & - & - & - & - & - & 456 & 100 \\
\hline
\end{tabular}

\section{Cuadro 11. Evolución clases léxicas romper $a+$ infinitivo}

Solo cuatro clases de las seis se observan en el corpus, a saber los verbos materiales, verbales, mentales y relacionales. Predomina claramente la clase léxica de los verbos materiales, como en el caso de ponerse/meterse, pero su frecuencia es aún más preponderante con un promedio de un $75 \%$ a lo largo de los tres siglos estudiados. Los infinitivos observados son: llorar, reír, lloriquear, sudar, regurgitar, toser, andar, correr, patinar, huir, hervir, cocer, bailar, llover, esgrimir, embestir (15). También la macroclase verbal es bastante frecuente, con un promedio de un 21,2\%, aunque parece perder terreno en el español actual. El corpus menciona los siguientes infinitivos: decir, escribir, publicar, explicar, gritar, callar y charlar, ladrar, cantar, gruñir, berrear, aplaudir y vitorear (16). La categoría de los verbos mentales se restringe al único caso de leer (mental - percepción) (17).

(15) a. El crío rompe a lloriquear. (CREA: Roa Bastos A., 1960)

b. Martín dobla el diario, lo guarda en el bolsillo de la americana, y rompe a andar. (CREA: José Cela C., 1951)

c. déjense cocer cinco minutos, empezando a contarlos desde que el agua rompe de nuevo a hervir. (CREA: Mestayer de Echagüe M., 1940)

d. Nos hicieron ir a los coches y nos juntamos todos, muy prietos, debajo de la marquesina, porque rompió a llover a cántaros. (CREA: Sánchez Mazas R., 1956) 
(16) a. Al oír que el cabo Ayuso preparaba su fusil ametrallador, uno de ellos se tiró al suelo mientras otro rompía a gritar: “¡Cobardes, cobardes! ¡Asesinos! ¡Ase...!” (CREA: Gironella J.M., 1961)

b. Aturdido quedó Ibero ante la precoz erudición que su amigo echó sobre él apenas rompieron a charlar. (CORDE: Pérez Galdós B., 1907)

c. Apoyado un hombro en la jamba de la puerta, a la expectativa de que rompiese a gruñir la cafetera, [...]. (CREA: García Hortelano J., 1972)

d. Una voz que fue como la llave que abrió las puertas al entusiasmo, ya que la gente, sin consideración a la excelente versión del tango que la rondalla ofrecía y casi ofrendaba, rompió a aplaudir y a vitorear al recién llegado. (CREA: Torrente Ballester G., 1972)

(17) Anselmo del Alamo permanecía callado esperando su turno para leer su poema, pero acto seguido Olalla Guzmán tomó el relevo, abrió su cuaderno y rompió a leer un interminable romance de rimas esdrújulas. (CORPES XXI: González Moreno P., 2007)

En conclusión, por un lado, los datos permiten negar claramente la idea aceptada que la perífrasis con romper se restringe a los verbos llorar y reír. El análisis de su productividad léxica confirma que gradualmente incluye más tipos infinitivales diferentes. Por el otro lado, tampoco nos pueden engañar estas conclusiones dado que los nuevos tipos pertenecen a una clase léxica determinada, a saber los verbos materiales (tipo 2).

\section{CONCLUSIÓN: PERÍFRASIS INCOATIVAS SINÓNIMAS, ¿FENÓMENOS DE CAMBIO IDÉNTICOS?}

El presente estudio de caso se ha propuesto arrojar luz sobre la evolución histórica de diferentes perífrasis incoativas cuasi-sinónimas en español. Su propósito no solo ha sido examinar su desarrollo formal y funcional, sino también determinar hasta qué punto los datos encajan en diferentes teorías del cambio lingüístico, en concreto los modelos de lexicalización y construccionalizacion.

Ante todo, ha llamado la atención la aparición tardía de la perífrasis romper a + infinitivo en la historia del español. Mientras que los bancos de datos proporcionan ejemplos de ponerse/meterse + infinitivo ya desde los primeros documentos del español, el primer texto que documenta romper a + infinitivo es de mediados del siglo XIX. Este solo hecho ha tenido consecuencias importantes para el análisis morfosintáctico subsiguiente: no se observan las alternancias preposicionales observadas en los corpus de las perífrasis incoativas con verbos de colocación, y mucho menos las estructuras no incorporadas. Por lo tanto nos parece lícito formular la hipótesis de que la perífrasis incoativa se ha desarrollado como construcción entre los siglos XIII y XVII con los verbos de colocación ponerse y meterse como núcleo, y que después de esta fase, una vez fijado el esquema, se abre a incorporar más verbos diferentes en la posición del verbo auxiliar.

Luego, el análisis de los rasgos léxico-semánticos de las perífrasis cuasi-sinónimas aclara su desarrollo posterior. En general, tanto en cuanto a los rasgos semánticos de la construcción entera como en cuanto a la clasificación aspectual y léxica del infinitivo subordinado, las perífrasis con ponerse/meterse se muestran más variadas y productivas que la variante con romper. Sin embargo, los datos no confirman la hipótesis de una fuerte lexicalización de romper a + infinitivo en el sentido de que se limitaría a unos pocos 
verbos auxiliados como llorar y reír. Su productividad en términos de tipos léxicos diferentes es mucho más alta, aunque sí casi se restringe a la sola clase de verbos materiales que denotan procesos. Además, los datos muestran que la productividad de las perífrasis con ponerse/meterse ha superado su clímax pero que se mantiene bastante estable en los últimos siglos.

En conclusión, el desarrollo de las perífrasis cuasi-sinónimas con ponerse y meterse por un lado, y romper por otro lado, se describe solo parcialmente mediante los mismos modelos: la construccionalización ha empezado con los verbos de colocación, pero abre el camino a la incorporación del verbo de cambio romper. La lexicalización se aplica solo al caso de romper, aunque nos parece que este proceso ha sido fuertemente contrapesado por la evolución general del esquema incoativo, y que no llega al nivel que se le asigna en la literatura.

\section{Referencias bibliográficas}

ADESSE corpus. Base de datos de verbos, alternancias de diátesis y esquemas sintáctico semánticos del español: http://adesse.uvigo.es/ (ADESSE) (09-01-2018).

Albertuz, F. J. (2007). "Sintaxis, semántica y clases de verbos: clasificación verbal en el proyecto ADESSE". En Actas del VI Congreso de Lingüística General (Santiago de Compostela, mayo 2004). Madrid: Arco Libros, pp. 2015-2030.

Aparicio, J., Castellón, I., y M. Coll-Florit (2011). "Rasgos y clases para la representación de la estructura aspectual", Anuari de Filologia. Estudis de Lingüistica, 1, pp. 1-29.

Aparicio, J., Castellón, I., y M. Coll-Florit (2014). "Perífrasis incoativas: aproximación cognitiva y estudio de corpus", Sintagma, 26, pp. 73-88.

Barðdal, J. 2008. Productivity: Evidence from case and argument structure in Icelandic. Amsterdam/ Philadelphia: John Benjamins.

Bergs, A., y G. Diewald (eds.) (2008). Constructions and language change. Berlin: Mouton de Gruyter.

Borillo, A. (2005). "Peut-on identifier et caractériser les formes lexicales de l'aspect en français?". En Bat-Zeev Shyldkrot H. y N. Le Querler (eds.). Les Périphrases Verbales. Amsterdam/Philadelphia: John Benjamins, pp. 83-102.

Brinton, L. y Traugott, E. (2005). Lexicalization and language change. Cambridge: University Press. Bybee, J., y R. Torres Cacoullos. (2009). "The role of prefabs in grammaticization." En Corrigan R., Moravcsik E., Ouali H. y K. Wheatley (eds.). Formulaic Language. Amsterdam/Philadelphia: John Benjamins, pp. 187-217.

Carrasco Gutiérrez, A., Camus Bergareche, B., Martínez-Atienza, M., García García-Serrano, A. y L. García Fernández (dirs.) (2006). Diccionario de perifrasis verbales. Madrid: Gredos.

Comer, M., y R. Enghels (2017). "La evolución de las perífrasis verbales causativa e incoativa con poner en español: cambios de construcción y extensiones metafóricas", Bulletin of Hispanic Studies, 94 (9), pp. 903-924.

Corominas, J. (1954). Diccionario crítico etimológico de la lengua castellaña. Berna: Editorial Francke.

Coussé, E. (2018). “Grammaticalization, host-class expansion and category change.” En van Goethem, K., Norde, M., Coussé, E. y G. Vanderbauwhede (eds.). Category change from a construction grammar perspective. Amsterdam/Philadelphia: John Benjamins, pp. 93-117.

Croft, W. (2001). Radical construction grammar: syntactic theory in typological perspective. Oxford: Oxford University Press.

Enghels, R. (2007). Les Modalités de perception visuelle et auditive: différences conceptuelles et répercussions sémantico-syntaxiques en espagnol et en français. Tübingen: Niemeyer. 
Enghels, R. y M. Comer (2018). "Evaluating grammaticalization and constructional accounts: the development of the inchoative construction with put verbs in Spanish". En Coussé E., Andersson P. y J. Olofsson (eds.). Grammaticalization meets construction grammar. Amsterdam/Berlin: John Benjamins, pp. 109-135.

Enghels, R. y M. Comer (en prensa). "Causative and Inchoative constructions with poner and meter ('to put') in Spanish : a diachronic constructional approach". En Fernández Jaen J. y H. Provencio Garrigós (eds.). Historical linguistics : current theories and applications editor. Amsterdam/ Berlin: John Benjamins.

Fauconnier, G. (1983). “Generalized Union.” En Tasmowski L. y D. Willems (eds.). Problems in syntax. Gent: Plenum, pp. 195-229.

Fischer, O. (2007). Morphosyntactic change. Functional and Formal Perspectives. New York: Oxford University Press.

Fogsgaard, L. (2001). "Algunas perífrasis incoativas con a + infinitivo", Estudios De Lingüistica: Universidad De Alicante, 15, pp. 247-260.

Garachana Camarero, M. (2011). "Perífrasis sinónimas: ¿gramaticalizaciones idénticas?”. En Bustos Tovar J., Cano Aguilar R., Méndez García de Pareded E. y A. López Serena (eds.). Homenaje a Antonio Narbona. Sevilla: Universidad de Sevilla, pp. 779-795.

Garachana Camarero, M. (2016). "Restricciones léxicas en la gramaticalización de las perífrasis verbales," RILCE, 32 (1), pp. 136-158.

Goldberg, A. (1995). Constructions: a construction grammar approach to argument structure. Chicago: University of Chicago Press.

Goldberg, A. (2006). Constructions at work: The nature of generalization in language. Oxford: Oxford University Press.

Gómez Manzano, P. (1992). Perifrasis verbales con infinitivo (valores y usos en la lengua hablada). Madrid: UNED.

Gómez Torrego, L. (1988). Perifrasis verbales. Madrid: Arco Libros.

Gómez Torrego, L. (1999). "Los verbos auxiliares. Las perífrasis verbales de infinitivo”. En Bosque, I. y V. Demonte (dirs.). Gramática Descriptiva de la Lengua Española. Madrid: Espasa Calpe, pp. 1209-1273.

García González, J. (1992). Perifrasis verbales. Madrid: Sociedad general española de librería.

Hilpert, M. (2008). Germanic Future Constructions. A usage-based approach to language change. Amsterdam: John Benjamins.

Hilpert, M. (2013). Constructional Change in English. Cambridge: Cambridge University Press.

Himmelmann, N. (2004). "Lexicalization and grammaticalization: opposite or orthogonal?" En Bisang W., Himmelmann N. y B. Wiemer (eds.). What Makes Grammaticalization - A Look from its Fringes and Its Components. Berlin: Mouton de Gruyter, pp. 19-40.

Hernanz, Lluïsa M. (1999). "El infinitivo". En Bosque I. y V. Demonte (dirs.). Gramática Descriptiva de la Lengua Española. Madrid: Espasa Calpe, pp. 2197-2356.

Lamiroy, B. y W. De Mulder (2011). "Degrees of Grammaticalization Across Languages". En Narrog H. y B. Heine (eds.). The Oxford handbook of grammaticalization. Oxford: Oxford University Press, pp. 302-317.

Lehmann, C. (2002). "New reflections on grammaticalization and lexicalization". En Wischer I. \& G. Diewald (eds.). New Reflections on Grammaticalization. Amsterdam: John Benjamins, pp. 1-18.

Lehmann, C. (2004). "Theory and method in grammaticalization". En Diewald G. (ed.). Grammatikalisierung. Special issue of Zeitschrift für Germanistische Linguistik, 32, pp. 152-187.

Moliner, M. (1998). Diccionario de uso del español. Madrid: Gredos. $2^{\text {a }}$ edición.

Noël, D. (2007). "Diachronic construction grammar and grammaticalization theory," Functions of language, 14 (2), pp. 177-202.

Real Academia Española. Diccionario de La Lengua Española (http://dle.rae.es/) (9/01/2018).

Traugott, E. y Trousdale, G. (2013). Constructionalization and constructional change. Oxford: Oxford University Press 
Trousdale, G. (2008). "Constructions in grammaticalization and lexicalization. Evidence from the history of a composite predicate construction in English." En Trousdale G. y N. Gisborne (eds.). Constructional approaches to English grammar. Berlin: Mouton de Gruyter, pp. 33-67.

Trousdale, G. (2014). "On the relationship between grammaticalization and constructionalization." Folia Linguistica, 48 (2), pp. 557-577.

Väänänen, V. (1968). Introduction au latin vulgaire. Paris: Klincksieck.

Vendler, Z. (1967). Linguistics in philosophy. Ithaca: Cornell university press.

Verroens, F. (2011). La construction inchoative se mettre à: syntaxe, sémantique et grammaticalisation. Tesis doctoral, Ghent University. 